\title{
Adaptive Redundant Residue Number System Coded Multicarrier Modulation
}

\author{
Thomas Keller, T. H. Liew, and Lajos Hanzo, Senior Member, IEEE
}

\begin{abstract}
The novel class of nonbinary maximum minimum distance redundant residue number system (RRNS) codes is invoked in the context of adaptively RRNS coded, symbol-by-symbol adaptive multicarrier modulation, in order to combat the effects of frequency-selective fading inflicted by dispersive wide-band channels. The system's performance can be adjusted in order to maintain a given target bit error rate (BER) and bit per symbol (BPS) performance. The proposed adaptive RRNS scheme outperforms the convolutional constituent code based turbo coded benchmarker system for channel signal-to-noise ratios (SNR) in excess of about $15 \mathrm{~dB}$ at a target BER of $10^{-4}$.
\end{abstract}

Index Terms-Adaptive channel coding, adaptive modulation, adaptive OFDM, coded multicarrier modulation, OFDM, redundant residue number system.

\section{INTRODUCTION}

\section{A. Advances in Multicarrier Modulation}

$\mathbf{T}$ HE PRINCIPLE of orthogonal frequency division multiplexing (OFDM) - which is also often referred to as multicarrier modulation-originates from Chang [1]. We first provide a brief historical perspective on OFDM research before we focus our attention on the research topic of the paper in the next subsection. Since its conception, generations of researchers have investigated this technique [2]-[11], and during the past decade, it has reached maturity, justifying its employment in a variety of practical systems. For example, the European digital audio broadcasting (DAB) standard [12], the digital terrestrial television broadcast (DTTB) system (now known as DVBT [13]), and a range of other high-rate applications, such as $155 \mathrm{Mb} / \mathrm{s}$ wireless asynchronous transfer mode (ATM) local area networks have opted for employing OFDM. Another important OFDM application is constituted by the so-called high performance local area network standard known as HIPERLAN, which a member of the broadband radio access networks family often referred to as BRAN [14], [15]. These wide-ranging applications underline its significance as an alternative technique to conventional channel equalization in order to combat signal dispersion [7]-[10], [16], [17].

A range of recent advances in OFDM are presented in the impressive state-of-art collection of works edited by Fazel and Fettweis [16]. The well-known crest-factor (CF) problem was addressed, for example, in [18]. Relying on the seminal idea of Kalet [11], Schmidt and Kammeyer [19] employed adaptive subcarrier allocation in order to reduce the crest factor. Dinis and

Manuscript received September 23, 1999; revised May 4, 2000. The authors are with the Department of Electronics and Computer Science, University of Southampton, SO17 1BJ, U.K. (e-mail: 1h@ecs.soton.ac.uk). Publisher Item Identifier S 0733-8716(00)09204-0.
Gusmão [20]-[22] researched the use of two-branch amplifiers, while the clustered OFDM technique introduced by Daneshrad, Cimini, and Carloni [23] invokes a set of parallel partial FFT processors. OFDM systems with increased robustness to nonlinear distortion have been proposed by Okada, Nishijima, and Komaki [24] as well as by Dinis and Gusmão [25]. Fine frequency and timing tracking algorithms exploiting the OFDM signal's cyclic extension were published by Moose [26], Daffara [27], and Sandell [28]. Combining adaptive antenna techniques with OFDM transmissions was shown to be advantageous in suppressing co-channel interference in cellular communications systems. Li, Cimini, and Sollenberger [29]-[31]; Kim, Choi, and Cho [32]; Lin, Cimini, and Chuang [33]; as well as Münster et al. [34] have investigated algorithms for multiuser channel estimation and interference suppression.

\section{B. Adaptive Multicarrier Modulation}

Against this background, we propose here channel-quality dependent variable rate channel coding schemes in conjunction with a symbol-by-symbol adaptive OFDM (AOFDM) arrangement, where the modulation mode of groups subcarriers can be regularly updated according to the instantaneous channel conditions, in order to improve the system's performance. More explicitly, in addition to the subcarrier modulation modes [11], [35]-[38], the coding rate is adapted in response to the time- and frequency-dependent estimated channel conditions. Burst-byburst AOFDM exploits the time-varying nature of the wireless communications channel, invoking the following modem functions [35]:

- channel quality estimation,

- choice of the appropriate modulation mode for each subcarrier, and

- either explicit signaling or inferring the modulation modes by invoking blind mode detection.

Since the modem parameter adaptation is tailored to the instantaneous channel conditions between the transmitter and the receiver, the proposed technique is essentially limited to point-to-point duplex communications. The perceived channel quality estimate of the receiver-or the set of corresponding modulation parameters - has to be communicated to the transmitter in order to invoke the appropriate modem mode for its transmission, which results in the required receiver target performance. More explicitly, the requested set of modulation and channel coding parameters to be used for the next AOFDM symbol has to be signaled by the receiver of user A to the remote transmitter of user B by superimposing this side-information on the transmitted AOFDM symbol of user A. However, this introduces channel quality estimation latency, an 


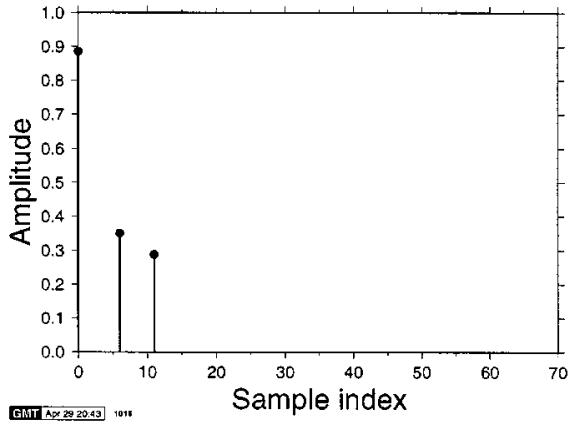

(a) $h(n)$
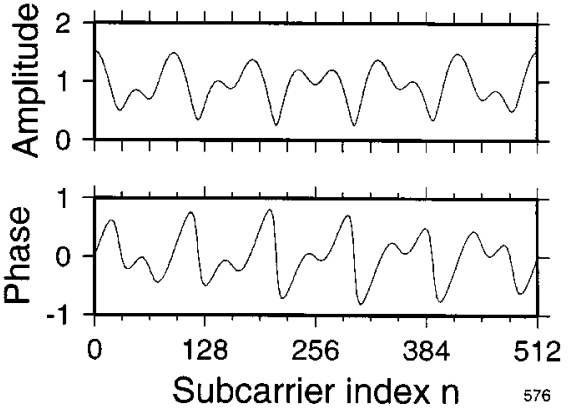

(b) $H_{n}$

Fig. 1. Wideband channel: (a) unfaded time-domain OFDM-sample-spaced impulse response and (b) corresponding frequency domain channel transfer function.

issue that was treated in the context of single-carrier modems in [39]. The channel quality estimation can be performed by using the known pilots in pilot symbol assisted AOFDM modulation (PSAM). In this paper, we assumed perfect knowledge of the channel transfer function and perfect modulation mode detection, concentrating on the system's upper-bound performance. Our proposed AOFDM scheme invoked three different modulation modes, as well as "no transmission," on a subcarrier by subcarrier basis. Specifically, 0, 1, 2, and 4 bits-per-symbol (BPS) quadrature amplitude modulation (QAM) schemes [40]—corresponding to binary phase shift keying (BPSK), quaternary phase shift keying (QPSK), and 16-QAM were used. Blind modem mode detection was the topic of [35] and [38].

The channel impulse response (CIR) of Fig. 1 was employed in our simulations, which consisted of a three-path impulse response, with a maximal path delay of 11 samples, where each path was faded according to a Rayleigh distribution, using a normalized maximal Doppler frequency of $f_{d}^{\prime}=1.235 \cdot 10^{-5}$, 1 which corresponds to the channel experienced by a wireless asynchronous transfer mode (WATM) modem transmitting at a carrier frequency of $60 \mathrm{GHz}$ with a sampling rate of $225 \mathrm{MHz}$ and a vehicular velocity of $50 \mathrm{~km} / \mathrm{h}$. The CIR exhibits a root mean squared (rms) delay spread of $1.5276 \cdot 10^{-8} \mathrm{~s}$. Furthermore, the CIR taps were constant for the duration of each OFDM symbol and were readjusted at the end of the 512-subcarrier OFDM symbol according to the Doppler frequency. We referred to this as symbol-invariant fading. Finally, each OFDM symbol had a cyclic prefix of 64 time-domain samples. Let us now focus our attention on the rationale behind the choice of channel codecs.

\section{Choice of Error Correction Codes}

The behavior of AOFDM in conjunction with long block-length convolutional coding based turbo coding has been studied in [35]. We have seen that the error correction performance of the turbo decoder was high, resulting in output bit error rates of below $10^{-4}$ for input bit error rates of up to $1 \%$. The disadvantage of this recursive systematic convolution (RSC) coding based turbo codec was the long turbo-inter-

\footnotetext{
${ }^{1}$ Normalization was carried out with respect to the OFDM symbol duration, not the time-domain sample spacing.
}

leaving block length of 2000 bits, which was necessary for achieving a high turbo-coded performance, but prevented code rate adaptation on a short-term basis. This mitigated the achievable gains due to adaptive-rate coding and AOFDM. In other words, radically shortening the interleaving block length of turbo codes results in significantly reduced performance, as does additional puncturing of the code word for code rate adaptation.

Similarly to AOFDM modem mode adaptation, the adaptive error correction codec has to be able to vary its code rate according to the frequency dependent channel transfer function observed for the OFDM system in the time-dispersive channel, as exemplified by the CIR transfer function of Fig. 1. Ideally, the error correction capability of the code would be adjustable for each data bit's expected BER independently, but naturally this is unrealistic. For our experiments, short block length codes of less than 100 bits per code word were required, in order to allow flexible adaptation of code parameters, while delivering reasonable error protection for the data bits.

Following the above introductory notes, in Section II we provide a brief overview of redundant residue number system (RRNS) codes, which are proposed for our system, and highlight their basic properties. This section also portrays the RRNS coding performance over Gaussian channels using QPSK. Section III commences with the introduction of the adaptive RRNS (ARRNS) coding philosophy using a range of different-rate RRNS channel coding modes and then characterizes the ARRNS-coded QPSK/OFDM system's performance over a) Gaussian channels and b) dispersive fading channels using fixed-mode QPSK/OFDM modulation. Section IV develops this system further to an ARRNS/AOFDM scheme using both a range of RRNS coding modes as well as various AOFDM modulation modes, and characterizes its performance, before concluding in Section V. Let us now consider the proposed RRNS codes.

\section{Redundant Residue Number System Codes}

Residue number system (RNS) [41], [42] based algorithms have been studied in the context of digital filtering, spectral analysis, correlation and matrix operations, as well as in image processing [43]-[46]. Until quite recently, RRNSs have only been proposed for fault-tolerant processing of signals, for example, 
in digital arithmetics. RRNSs represent each operand with the aid of a set of residues. The residues are generated as the remainders upon dividing the operand to be represented with the aid of the residues by each of the so-called moduli of the RRNS, which have to be relative primes, i.e., do not have a common divisor. Among others, the following two advantages accrue for RRNS-based processing [47]. First, they have the ability to use carry-free arithmetic, since all residue-based operations can be processed independently of each other. Hence, no carry forward has to "ripple through" from the least to the most significant bits due to the lack of ordered significance among the residue digits. This property is amenable to high-speed parallel processing of the independent residues. Furthermore, the lack of ordered significance of the residue digits implies that as long as a sufficiently high number of residues is available, in order to unambiguously represent the results of the computations, any erroneous residue digit can be discarded without affecting the result.

Error detection and correction algorithms based on the RNS have been proposed by Szabo et al. [41], as well as by Watson and Hastings [42], which exploited the properties of the redundant residue number system (RRNS). More recently, a computationally efficient procedure was described in [48] for correcting a single error. In [49], the procedure was extended to correcting double errors as well as simultaneously correcting single and detecting multiple errors. Efficient soft-decision based multiple error correcting algorithms were suggested in [50]. Furthermore, an RNS-based $M$-ary modulation scheme has been proposed and analyzed in [51], while an RRNS-based CDMA system was the topic of [52].

$\operatorname{RRNS}(n, k)$ codes are akin to the well-known family of Reed-Solomon (RS) codes, and both of these codes achieve the so-called maximum minimum distance of $d=n-k+1$, provided that the moduli of the RRNS code obey certain conditions. It can also be readily shown that the RRNS code's weight-distribution can be approximated by the weight-distribution of RS codes, if all the moduli assume values close to their average value [49], [53], [54]. For further details concerning the construction, coding theory, encoding and decoding of RRNS codes, the interested reader is referred to [49]-[54].2 Based on the above arguments, a similar coding performance is achieved by an identical-rate RS-code and RRNS-code, provided that they both use the same number of bits per symbol or bits per residue. However, their encoding and decoding algorithms are distinctly different [50], requiring further research in implementational, coding theoretical and application terms. Since in [50] the authors have developed a soft-decision based decoding algorithm, here we favored RRNS codes, with the intention of stimulating further research in this interesting novel field. Hence, upon using the soft-decision algorithm of [50], the above code selection criteria in the context of RS codes would result in a similar performance to the RRNS codes used here [55].

The RRNS codes employed in our investigations are systematic, which means that $k$ of the $n$ code residues contain the original data bits, and the additional $(n-k)$ redundant residues can

\footnotetext{
${ }^{2} \mathrm{~A}$ range of related (but at the time of writing, unpublished) RRNS-coding oriented papers by the authors can be found under http://www-mobile.ecs.soton.ac.uk/lly/.
}

TABLE I

RRNS CODES EMPLOYED IN OUR INVESTIGATIONS, EACH USING 8-BIT RESIDUES

\begin{tabular}{l|r|r|r|r|r|r}
\hline Code & $(5,3)$ & $(7,3)$ & $(9,3)$ & $(8,6)$ & $(10,6)$ & $(12,6)$ \\
\hline Sys. residues & 3 & 3 & 3 & 6 & 6 & 6 \\
\hline Red. residues & 2 & 4 & 6 & 2 & 4 & 6 \\
\hline Correction capability $t$ & 1 & 2 & 3 & 1 & 2 & 3 \\
\hline Code rate & 0.6 & 0.43 & 0.33 & 0.75 & 0.6 & 0.5 \\
\hline Data bits per word & 24 & 24 & 24 & 48 & 48 & 48 \\
\hline Red. bits per word & 16 & 32 & 48 & 16 & 32 & 48 \\
\hline
\end{tabular}

be employed for error correction at the decoder. The error correction capability of the code is $t=\lfloor(n-k) / 2\rfloor$ residues [50].

The code rate, and accordingly the error correction capability of the code, can be readily varied by transmitting only a fraction of the generated redundant residues. If the channel conditions are favorable, then only the systematic information-bearing residues are transmitted, resulting in a unity-rate code with no added redundancy and no error correction capability. Upon transmitting two redundant residues along with the data-bearing residues, the resulting code can correct one residue error for a code rate of $n /(n+2)$. More of the redundant residues can be transmitted, lowering the code rate and improving the code's error resilience at the cost of a lower effective information throughput, when the channel quality degrades.

In our investigations, RRNS codes employing 8 bits per residue have been chosen. Three or six systematic information-bearing residues-corresponding to 24 or 48 useful data bits per code word-and up to six redundant residues have been employed. The code parameters for these codes are shown in Table I. A maximum of nine residues were necessary, for example, for the $\operatorname{RRNS}(9,3)$ code and relative prime moduli used were 229, 233, 239, 241, 247, 251, 253, 253, 255, and 256. The residues with respect to all these moduli were generated, and the required number of redundant residues were appended to the information bearing residues. Simple logic dictates that only the residue associated with the modulus of 256 can carry eight bits. However, in [50] a technique was proposed for mapping eight bits, rather than only seven bits, to those residues, which were between 128 and 256. In simple terms, the procedure of [50] may map two 8-bit tuples-which exhibit a high Hamming distance, such as, for example, 00000000 and 11111111 - to a residue between 128 and 256 and invokes the demodulator's soft outputs for deciding, which was the more likely transmitted 8-bit tuple. This technique was used to ensure that all residues were capable of carrying an 8-bit tuple, even when the RRNS decoder was employing hard-decision decoding. As can be seen from Table I, the code rates vary from 0.33 to 0.75 . The uncoded case, corresponding to a $(3,3)$ code, is not shown in the table.

The BER performance of an OFDM system employing QPSK and the RRNS codes of Table I is depicted in Fig. 2. It can be seen that the relative BER performance of the different codes is largely in line with their respective code rates. The $(9,3)$ code-with a code rate of 0.33 - exhibits the strongest error 


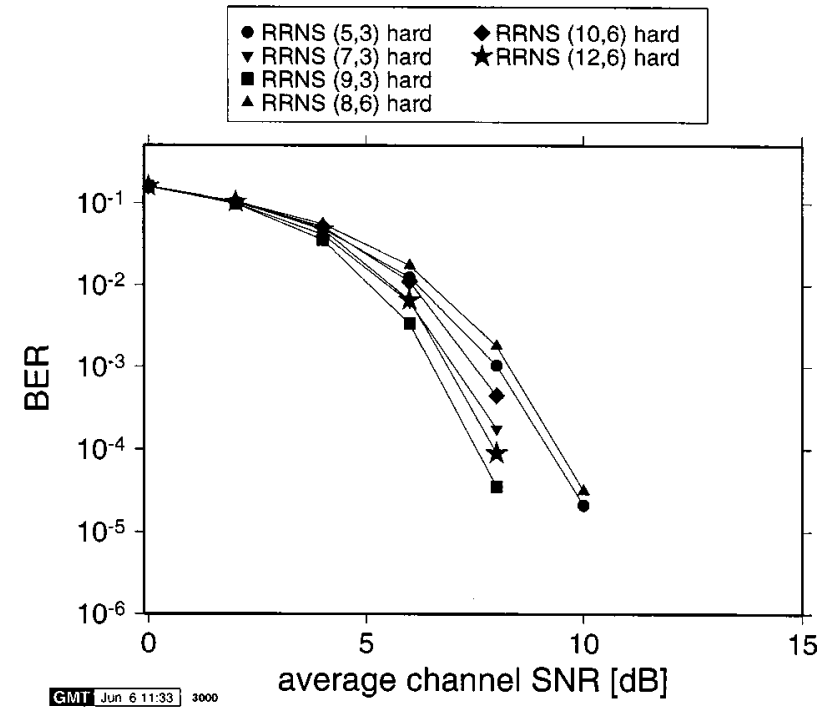

Fig. 2. BER performance for RRNS coded QPSK/OFDM transmissions in AWGN channels using hard-decision decoding and 8-bit residues.

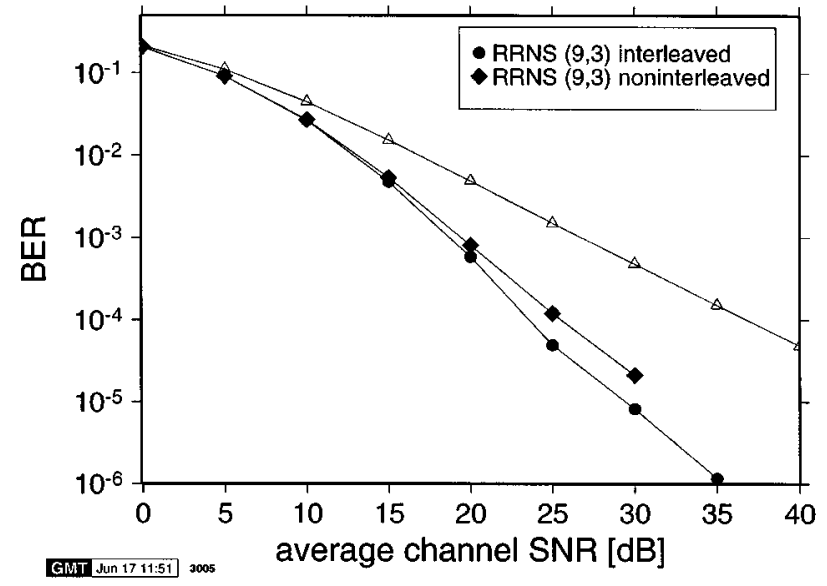

Fig. 3. BER performance for the RRNS(9, 3)-coded OFDM system in the Rayleigh fading time-dispersive WATM channel of Fig. 1 for 512-point FFT-based QPSK/OFDM transmission, portraying also the uncoded benchmarker performance.

correction properties; and the $(8,6)$ code-with a code rate of 0.75 - is the weakest code of the set. Comparing the performance of the $(5,3)$ code to that of the $(10,6)$ code - both having a code rate of 0.6 - shows that the longer code exhibits a superior performance. The $(12,6)$ code, having a code rate of 0.5 , outperforms the shorter $(7,3)$ code for SNR values in excess of $6 \mathrm{~dB}$.

Fig. 3 portrays the BER performance of a $\operatorname{RRNS}(9,3)$-coded OFDM modem using 512 subcarriers and an FFT-length of 512 (512-FFT) employing QPSK transmission over the Rayleigh fading time-dispersive channel. The associated BER curves for both uncoded as well as for coded noninterleaved and coded residue-interleaved transmission are shown. The interleaver employed in acquiring the interleaved results was a simple rectangular residue-based block-interleaver. This residue interleaver structure was chosen, since it is sufficiently flexible for varying numbers of residues, if adaptive modulation is invoked.

We note that residue-based interleaving, i.e., 8-bit symbol-based interleaving, has a better performance than
TABLE II

RRNS CODING MOdes UsEd FOR THE CODE RATE AdAPTATION EMPLOYING 8-BIT RESIDUES

\begin{tabular}{l|l|r|r|r}
\hline Mode $c$ & 0 & 1 & 2 & 3 \\
\hline$n_{c}$ & 3 & 5 & 7 & 9 \\
\hline$k_{c}$ & 3 & 3 & 3 & 3 \\
\hline$t_{c}$ & 0 & 1 & 2 & 3 \\
\hline$R$ & 1 & 0.6 & 0.43 & 0.33 \\
\hline
\end{tabular}

bit-interleaving, since bit-interleaving would increase the probability of residue errors due to spreading bursts of erroneous bits across residues. Since the RRNS decoding algorithm is symbol based [50], the increased residue error rate would degrade the system's performance.

It can be seen from Fig. 3 that the RRNS(9, 3)-coded schemes deliver a gain of about $12 \mathrm{~dB}$ in SNR terms at a BER of $10^{-4}$. Interleaving of the residues over the entire length of the OFDM symbol improves the BER performance by about $2 \mathrm{~dB}$ at this BER.

\section{ADAPTIVE RRNS CODED OFDM/QPSK}

\section{A. Adaptive RRNS Coding Philosophy}

Analogously to the AOFDM schemes discussed in the literature [35], [36], the code rate adaptation reacts to the time- and frequency-varying channel conditions experienced in a duplex link. Each communicating station exploits the channel quality information extracted from the most recent received OFDM symbol for determining the transmitter's coding parameters of the next transmitted frame, required for meeting its target integrity.

Although the longer RRNS codes exhibit better error correction properties than the shorter codes investigated, we will concentrate on the group of $(9,3),(7,3),(5,3)$ codes and on uncoded $(3,3)$ RRNS/OFDM transmission for the variable code rate application, since a short RRNS code word length allows for increased flexibility in terms of the adaptation to the channel conditions. These codes exhibit an error correction capability of $t=3,2,1$, and 0 residues per code word, respectively. Correspondingly, four error correction coding modes $c$ were defined, which are shown in Table II.

The choice of the coding mode for each RRNS code word in the OFDM symbol is determined on the basis of the estimated channel transfer function. As has been discussed in the context of the AOFDM scheme in [35], the predicted bit error probabilities $p_{e}$ are calculated for all bits to be transmitted in an OFDM symbol, based on the estimated subcarrier SNR and the modulation mode to be employed. More explicitly, the expected overall bit error probability for the given OFDM symbol is computed by averaging the estimated individual subcarrier BERs for all legitimate modulation modes $M_{n}$, yielding $\bar{p}_{e}\left(M_{n}\right)=$ $1 / N_{s} \sum_{j} p_{e}\left(\gamma_{j}, M_{n}\right)$, where $\gamma_{j}$ is the subcarrier SNR for $j=$ $1 \cdots 512$, and $N_{s}$ is the number of subcarriers used in the averaging process. The specific subcarrier modem mode allocations having the highest bits per symbol (BPS) throughput, whose 
estimated BER is lower than the required value, is then confirmed if AOFDM is used. This algorithm allows the direct adjustment of the desired maximum BER. At this stage, however, only QPSK modulation was invoked, which simplified the BER calculations, since only the coding mode was varied. AOFDM will be the topic of Section IV.

If OFDM is to be employed in conjunction with adaptive RRNS coding, then the number of bits per OFDM symbol and the mapping of bits to subcarriers can change from one OFDM symbol to the next. Explicitly, this implies using a variable bit rate, near-constant BER regime. Hence, the RRNS coding scheme adaptation algorithm operates on the basis of the estimated BER, rather than directly relying on the estimated channel transfer function. Once the vector of estimated bit error probabilities $p_{e}(n)$ for the specific number of bits $N_{b}$-which has to be conveyed by the OFDM symbol-is known, the total number of bits to be transmitted is split into blocks of $K=8$ bits, where $K$ is the number of bits per residue. Again, the error correction capability of the code in each RRNS code word is a given number of $K=8$-bit residues, not bits. Hence, as argued before, interleaving of bits would increase the residue error rate at the decoder's input, and hence it would reduce the system's performance.

From the bit error probability $p_{e}(n)$ of bit $n, n=1 \cdots N_{b}$, where $N_{b}$ is the number of bits allocated to the OFDM symbol, the estimated residue error rate $p_{r}(r)$ for residue index $r$, which is defined as the proportion of residues in error, for the $N_{r}=$ $\left\lfloor N_{b} / K\right\rfloor$ number of residues in the OFDM symbol can be calculated as

$$
p_{r}(r)=1-\prod_{n=0}^{K-1}\left(1-p_{e}(r \cdot K+n)\right) .
$$

The remaining $\left(N_{b}-K \cdot N_{r}\right)$ data bits of the OFDM symbol that are not allocated to any residue are filled with padding bits and hence contain no useful data. The mapping of the residues with index $r$ to the RRNS code words is based on the estimated residue error probabilities $p_{r}(r)$. An interleaver, $I(r)$, is used to map the stream of residues to the residue positions in the transmitted OFDM symbol.

A received RRNS code word of the codec mode $c_{w}$ is uncorrectable, if more than $t_{c}$ of the received residues are in error. The RRNS code word error probability $p_{w}$ for word $w$ can be calculated as

$$
p_{w}(w)=p\left(R_{r}(w)>t_{c_{w}}\right)=1-P\left(R_{r}(w) \leq t_{c_{w}}\right)
$$

where $R_{r}(w)$ is the number of residue errors in code word $w$, and $p_{w}(w)$ can be calculated from the residue error probabilities $p_{r}(r)$ as

$$
\begin{gathered}
p_{w}(w)=1-p\left[R_{r}(w)=0\right]-p\left[R_{r}(w)=1\right] \\
-\cdots-p\left[R_{r}(w)=t_{c_{w}}\right] .
\end{gathered}
$$

Upon elaborating further:

$$
\begin{aligned}
p & \left(R_{r}(w)=0\right) \\
\quad & =\prod_{r=0}^{n_{c_{w}}-1}\left(1-p_{r}\left(I\left(r_{0, w}+r\right)\right)\right)
\end{aligned}
$$

$$
\begin{aligned}
& p\left(R_{r}(w)=1\right) \\
& =\sum_{r=0}^{n_{c_{w}}-1} p_{r}\left(I\left(r_{0, w}+r\right) \prod_{s=0, s \neq r}^{n_{c_{w},}-1}\left(1-p_{r}\left(I\left(r_{0, w}+s\right)\right)\right)\right. \\
& =p\left(R_{r}(w)=0\right) \cdot \sum_{r=0}^{n_{c_{w}}-1} \frac{p_{r}\left(I\left(r_{0, w}+r\right)\right)}{1-p_{r}\left(I\left(r_{0, w}+r\right)\right)} \\
& p\left(R_{r}(w)=2\right) \\
& =\frac{1}{2} \cdot p\left(R_{r}(w)=0\right) \cdot \sum_{r=0}^{n_{c_{w}}-1}\left[\frac{p_{r}\left(I\left(r_{0, w}+r\right)\right)}{1-p_{r}\left(I\left(r_{0, w}+r\right)\right)}\right. \\
& \left.\cdot \sum_{s=0, s \neq r}^{n_{c_{w}}-1} \frac{p_{r}\left(I\left(r_{0, w}+s\right)\right)}{1-p_{r}\left(I\left(r_{0, w}+s\right)\right)}\right] \\
& p\left(R_{r}(w)=3\right) \\
& =\frac{1}{3 !} \cdot p\left(R_{r}(w)=0\right) \cdot \sum_{r=0}^{n_{c w}-1}\left[\frac{p_{r}\left(I\left(r_{0, w}+r\right)\right)}{1-p_{r}\left(I\left(r_{0, w}+r\right)\right)}\right. \\
& \cdot \sum_{s=0, s \neq r}^{n_{c w}-1}\left[\frac{p_{r}\left(I\left(r_{0, w}+s\right)\right)}{1-p_{r}\left(I\left(r_{0, w}+s\right)\right)}\right. \\
& \left.\left.\cdot \sum_{t=0, t \neq q, r}^{n_{c_{w}}-1} \frac{p_{r}\left(I\left(r_{0, w}+t\right)\right)}{1-p_{r}\left(I\left(r_{0, w}+t\right)\right)}\right]\right]
\end{aligned}
$$

where $r_{0, w}$ is the index of the first residue in code word $w$.

The RRNS codeword allocation is based on the following algorithm. The code rate adaptation algorithm calculates the word error probability $p_{w}(w)$ for the RRNS code word index $w$ in conjunction with the lowest-power RRNS codec mode of Table II, i.e., for $c=0$. If the word error probability exceeds a certain threshold of $p_{w}(w)>\alpha$ for $c=0$, then the next stronger coding mode, i.e., $c=1$, is selected, and the word error probability is evaluated again. If the new RRNS code word error probability (WEP), or synonymously the word error rate (WER), exceeds the threshold $\alpha$, then again, the next codec mode is evaluated until the estimated RRNS code word error probability falls below the threshold $\alpha$, or until the highest-power codec mode is selected. The WER threshold $\alpha$ can be used to control the adaptation process.

\section{B. Adaptive RRNS/Fixed-Mode OFDM for the AWGN Channel}

The effect of different values of the threshold parameter $\alpha$ on the effective data throughput and bit error rate (BER) of a QPSK modem in an AWGN channel is shown in Fig. 4. The word error rate (WER) threshold $\alpha$ was varied from $10^{-1}$ up to $10^{-4}$, and it can be seen that the system performance varies only in a limited range of SNR values-approximately between 7 and $12 \mathrm{~dB}$ - with the WER $\alpha$. The three coded BER curves of Fig. 2 are easily identified in this figure as the corresponding segments of the adaptive scheme's BER performance curve along with the BER curve of uncoded QPSK. The different codec modes are easily identifiable in this figure, since in the AWGN channel the channel quality is time-invariant, and therefore the same codec mode is chosen for all transmitted RRNS code words at a given SNR value. The choice of the RRNS codec mode 


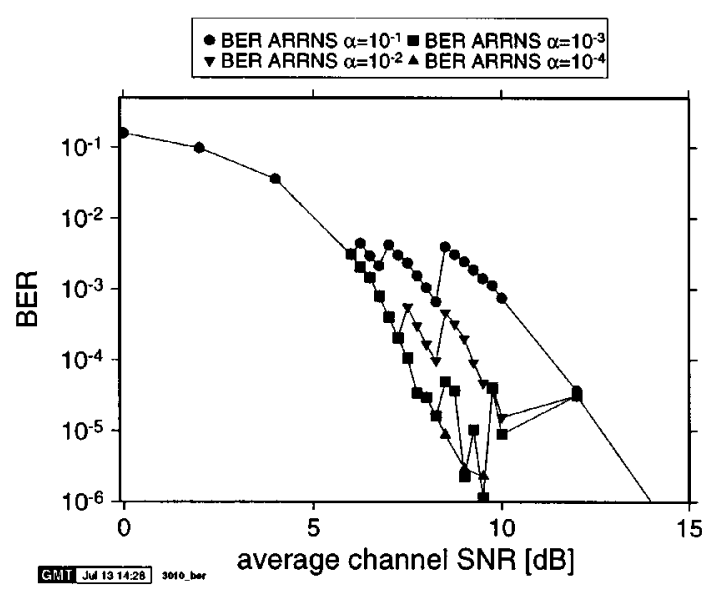

(a) BER

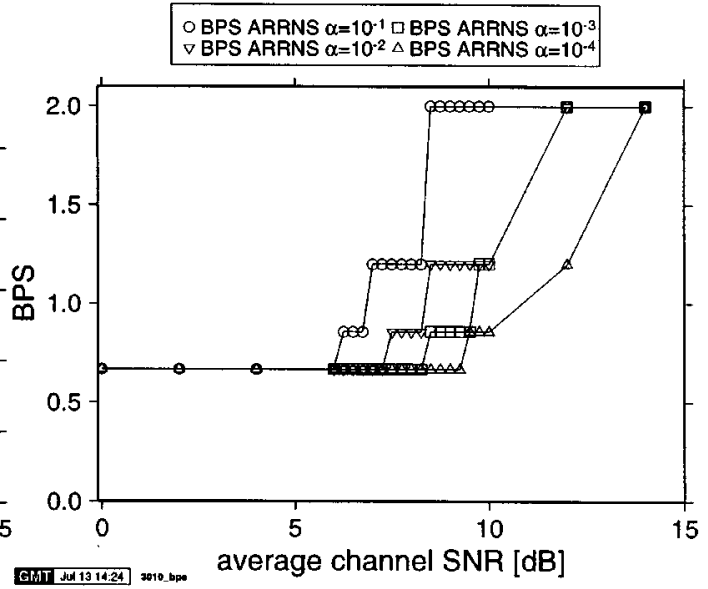

(b) BPS throughput

Fig. 4. BER performance and throughput in bits per (BPS) symbol for the Adaptive RRNS (ARRNS) coded OFDM system in AWGN channels, with QPSK transmission in the subcarriers.

clearly depends on the value of the WER $\alpha$. For higher SNR values, it can be observed that the WER $\alpha$ influences the codec mode switching algorithm. For $\alpha=10^{-1}$, the codec mode is switched to the next lower-power, higher-rate mode of Table II at SNR values of $6.25,7$, and $8.5 \mathrm{~dB}$, resulting in a BER of about $0.7 \%$ after each reconfiguration as seen in Fig. 4. The maximal throughput of 2 bits/symbol associated with uncoded QPSK is reached at an SNR of $8.5 \mathrm{~dB}$ by the $\alpha=10^{-1}$ curve. For lower values of $\alpha$, the codec mode switching is more conservative, and hence lower bit error rates and lower throughputs are observed. For $\alpha=10^{-4}$ the modem employs codec mode 3 of Table II for SNR values of up to $9.5 \mathrm{~dB}$, reaching mode 0 at $14 \mathrm{~dB}$ SNR.

\section{ARRNS/OFDM for Dispersive Fading Channels}

Over fading time-dispersive channels, the ARRNS codec mode is adapted to the time- and frequency-variant channel conditions. In order to demonstrate the RRNS code allocation process, in Fig. 5 we captured the expected subcarrier SNR versus the subcarrier index for one specific OFDM symbol. It can be seen that the channel SNR varies by about $18 \mathrm{~dB}$ across the set of subcarriers, which will result in a varying BER across the OFDM symbol. In our illustrative example, a specific OFDM symbol was selected that contained at least one RRNS code word corresponding to each of the RRNS codec modes of Table II. In order to augment our understanding, in this example, the residues of only one RRNS code word per codec mode are shown in the figure, corresponding to a total of $3+5+7+9=24$ residues, signified by vertical bars in the figure. Each vertical bar corresponds to one transmitted residue, and the gray shade of each bar relates it to one of the four different-rate ARRNS code words. The position of a bar gives the residue index $r$, while its height signifies the expected residue error rate (RER) $p_{r}(r)$ for each of the residues of the four chosen ARRNS code words, which depends on the subcarrier SNR experienced at index $r$.

The channel model used for the fading experiments was a three-path Rayleigh fading channel with a normalized Doppler

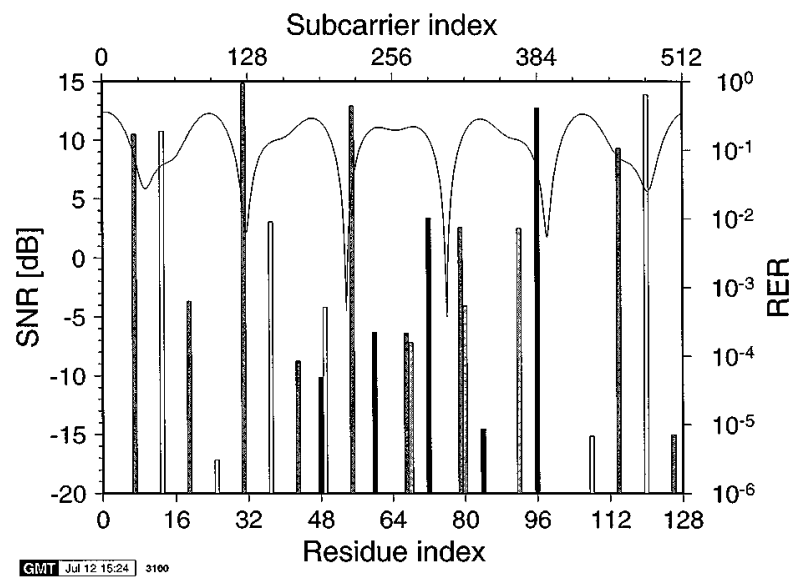

Fig. 5. Predicted subcarrier SNR (continuous line) for an given OFDM symbol and the associated residue error probabilities (vertical bars) for four selected ARRNS code words for a 512-subcarrier OFDM modem employing QPSK over the Rayleigh-fading time-dispersive WATM channel of Fig. 1. The average SNR is $10 \mathrm{~dB}$, and $\alpha=10^{-1}$. Key: light-grey- $(3,3)$ code at indices of $68,80,92$; black- $(5,3)$ at $48,60,72,84,96$; white- $(7,3)$; dark-grey $(9,3)$

frequency of $1.235 \cdot 10^{-5}$, as seen in Fig. 1 . The word error probability threshold $\alpha$ was set to $10^{-1}$.

In our example of Fig. 5, the light gray bars at residue indices 68,80 , and 92 indicate the residue error probabilities for an uncoded (mode 0) $(3,3)$ RRNS code word. The positions of the residues in the transmitted OFDM symbol are determined by the residue-based interleaver function. It can be seen that, because of the good channel quality in the mode 0-related OFDM subcarriers used for transmission, all of the three residues of the uncoded code word exhibit low RER values even without channel coding. The word error probability for this $(3,3)$ code word is mainly dominated by the highest RER residue transmitted at index 92 , which exhibits an error probability of about $7.2 \cdot 10^{-3}$. The resulting word error probability due to all three residues is $7.9 \cdot 10^{-3}$, which is below the threshold of $\alpha=10^{-1}$, and therefore uncoded transmission was chosen by the algorithm for the corresponding OFDM subcarriers. 


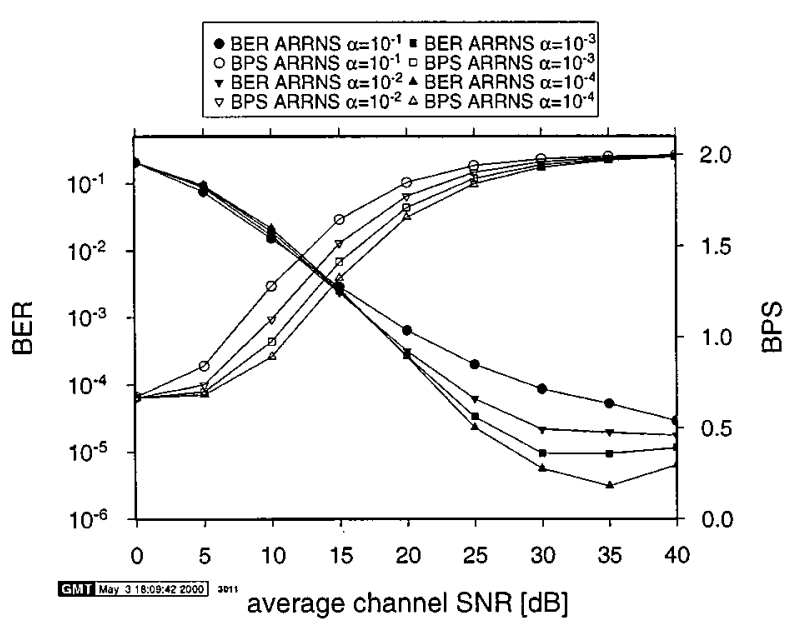

(a) non-interleaved

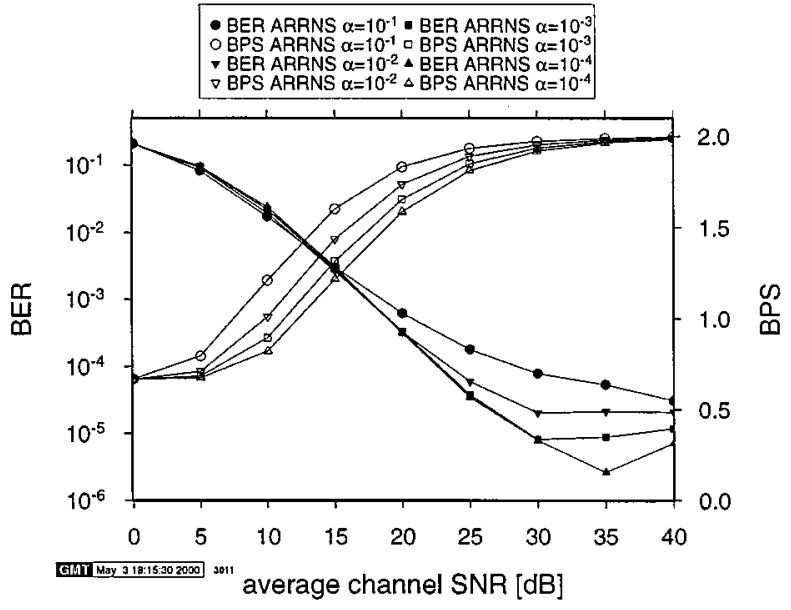

(b) interleaved

Fig. 6. BER and BPS throughput versus average channel SNR for ARRNS coded 512-subcarrier OFDM transmission employing QPSK over the Rayleigh fading time-dispersive WATM channel of Fig. 1 . The stipulated WER values were $\alpha=10^{-1}, 10^{-2}, 10^{-3}$, and $10^{-4}$.

Similarly, the black bars at residue indices of $48,60,72,84$, and 96 in Fig. 5 mark the residues for a mode $1(5,3)$ code word, with five transmitted residues, while the white marked residues form a mode $2(7,3)$ code word, consisting of seven transmitted residues. The first of this code word's residues at residue index $r=0$ is not visible on the figure, since its expected RER is $2.1 \cdot 10^{-7}$. The dark gray bars of Fig. 5, finally, represent a mode $3(9,3)$ RRNS code word with 9 transmitted residues. The word error probability for this mode 3 RRNS code word is $11.5 \%$, which exceeds the set threshold. Since there is no stronger code in the set of coder modes of Table II, the lowest possible code rate associated with the $\operatorname{RRNS}(9,3)$ code was chosen.

Fig. 6 shows the BER performance and data throughput of a 512-subcarrier ARRNS coded OFDM modem employing QPSK over the fading time-dispersive channel of Fig. 1. Specifically, Fig. 6(a) depicts the BER and BPS performance for the modem employing no residue-interleaving, while in the context of Fig. 6(b) a conventional rectangular residue-interleaver was employed. This comparison is relevant and necessary, since it is not intuitive, as to whether residue-interleaving results in performance improvements. This is because the residue-interleaving disperses the bursty channel errors across the OFDM subcarriers, which is expected to improve the coding performance of conventional fixed-rate coding. However, we proposed adaptive RRNS-coding in order to combat the bursty error distribution across the OFDM subcarriers, which was designed to counteract the frequency-selective fading and its bursty errors. Hence, interleaving and adaptive RRNS coding may have disadvantageously interfered with each other, necessitating a comparison of the interleaved and noninterleaved results.

It can be seen in Fig. 6 that the BER performance is fairly similar for the interleaved and noninterleaved modems. Specifically, although the noninterleaved modem slightly outperforms the interleaved one in BER terms for WERs of $\alpha=10^{-3}$ and for $\alpha=10^{-4}$ at SNR values in excess of $25 \mathrm{~dB}$, the BER difference is not significant. Upon comparing the achieved throughput, however, it is clear that the noninterleaved modem offers an average throughput benefit of about $0.1 \mathrm{bit} / \mathrm{symbol}$ for all target WERs $\alpha$ in the SNR region up to $25 \mathrm{~dB}$. Although not shown explicitly, similar findings were valid also for the AOFDM scenarios of the next section. Hence, due to space constraints, we only plotted results for the noninterleaved transceivers in the remainder of the paper. Since the range of RRNS code rates is limited, the BER performance at low values of SNR cannot be significantly lowered. Similarly to the AOFDM systems of [35], transmission blocking-defined as assigning zero bits to the low-quality AOFDM subband-would have to be introduced for the low-quality subcarriers, in order to guarantee a certain target bit error rate. Let us now consider the combination of ARRNS based coding and AOFDM modulation in the next section.

\section{ARRNS/AOFDM TRANSCEIVERS}

In this section we will demonstrate that upon combining AOFDM with adaptive RRNS coding, the low-SNR performance can be dramatically improved by amalgamating transmission blocking for the low-quality subcarriers with adaptive error correction coding. We have advocated here the target-BER adaptive modulation algorithm of [35] due to its high performance and adjustability to different target bit error rates.

The transmission parameter adaptation is performed in two steps. First, the AOFDM modulation modes are allocated to the subcarriers according to the algorithm outlined in [35]. Following this step, the number of bits $N_{b}$ to be transmitted in the next OFDM symbol and their estimated bit error probabilities $p_{e}(n)$, are known. On the basis of this, the code rate adaptation algorithm calculates the residue error rates $p_{r}(r)$ from (1), constructs the interleaver $I(r)$ for the correct number of residues, and invokes the appropriate codec modes for the RRNS code words, as outlined above. 


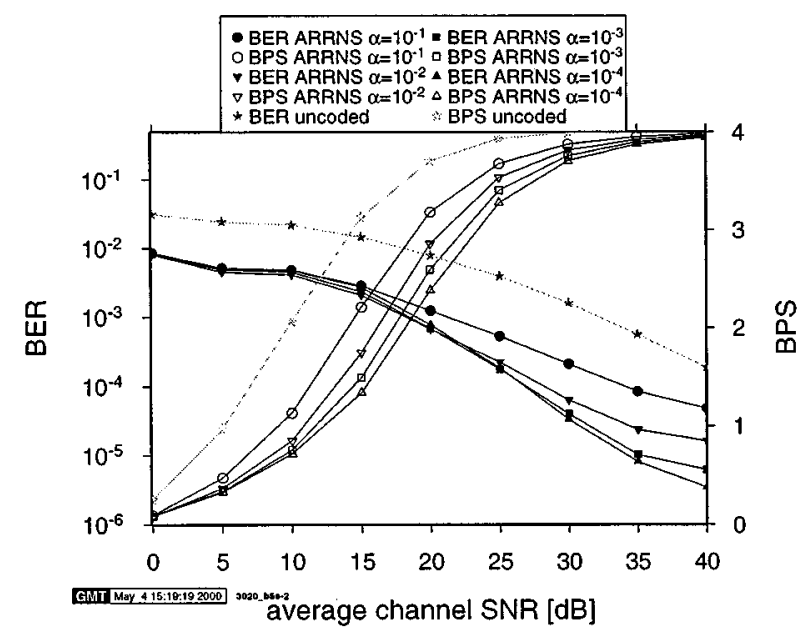

(a) target BER 5\% non-interleaved

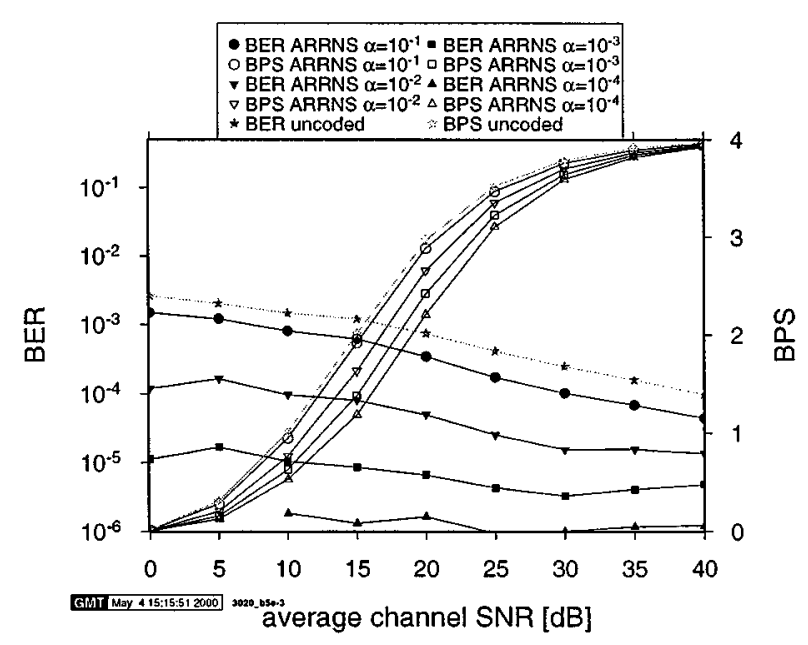

(b) target BER $0.5 \%$ non-interleaved

Fig. 7. BER and BPS throughput versus average channel SNR for hard-decision ARRNS-coded 512-subcarrier OFDM transmission employing adaptive modulation over the Rayleigh fading time-dispersive WATM channel of Fig. 1. (a) Uncoded adaptive modulation target BER 5\%. (b) Uncoded target BER 0.5\% The stipulated WER values were $\alpha=10^{-1}, 10^{-2}, 10^{-3}$, and $10^{-4}$. The light grey curves show the uncoded BER and BPS throughput.

Fig. 7 gives an overview of the ARRNS/AOFDM system's BER and throughput performance over the fading time-dispersive channel. Two target BER values have been stipulated, both with and without interleaving of the transmitted residues. Fig. 7(a) portrays the system's performance for an uncoded target BER of $5 \%$ for the ARRNS/AOFDM scheme without interleaving. It can be seen that the coded BER is then below $1 \%$ for all simulated ARRNS/AOFDM modem configurations, and that the SNR gain is much higher than for the fixed QPSK transmission of Fig. 3. The BER performance is limited, however, by the limited error correction capability of the RRNS $(9,3)$ mode when the SNR is very low.

Upon lowering the uncoded adaptive modulation's target BER to $0.5 \%$, the BER of the ARRNS/AOFDM system can be influenced over the whole SNR range by varying the WER $\alpha$. Fig. 7(b) depicts the corresponding BER and BPS throughput where: for a WER of $\alpha=10^{-1}$, the achieved ARRNS/AOFDM BER is better than $2 \cdot 10^{-3}$; for $\alpha=10^{-2}$ a BER of $2 \cdot 10^{-4}$, and for $\alpha=10^{-3}$ a BER of $2 \cdot 10^{-5}$ are never exceeded.

Comparing the interleaved performance to the noninterleaved results, it can be seen that the BER performance of comparable modems is fairly similar. The throughput is slightly higher, however, for the noninterleaved systems, demonstrating the efficiency of the hard-decision based ARRNS/AOFDM schemes in terms of combating the bursty errors of frequency-selective fading. Let us now consider the effects of invoking soft-decisions.

\section{A. Soft Decision RRNS Decoding}

The performance of the ARRNS codes can be increased by soft-decision decoding [50]. Previously, the ARRNS decoder assumed that the outputs of the demodulator were binary harddecision values. However, our RRNS decoder is capable of exploiting soft outputs provided by the demodulator at the receiver. Soft decoding of the ARRNS codes can be implemented by combining the classic Chase algorithm [56] with the hard decision based ARRNS decoder.

Fig. 8 shows the soft-decision decoded performance of the AOFDM/ARRNS system. Comparison to Fig. 7 shows an improved BER performance for the ARRNS soft decoder. This is especially significant for the target bit error rate of 5\%, where the soft-decoded AOFDM/ARRNS system achieves BER values of below $0.3 \%$ at $0 \mathrm{~dB}$ SNR. Since the adaptation algorithm is unchanged, the same throughput is observed for the hard- and soft-decoded systems. A BER of below $10^{-4}$ was registered for the $0.5 \%$ uncoded target BER system for a WER of $\alpha=10^{-2}$. Under these circumstances, the interleaved system exhibits a lower throughput and worse BER performance than the noninterleaved system.

\section{COMPARISON AND CONCLUSION}

In conclusion, Fig. 9 shows the throughput of the various ARRNS/AOFDM transmission systems studied for a channeldecoded target data BER of $10^{-4}$. The lightly shaded curves represent the variable throughput systems' performance graphs from [35]. The ARRNS/AOFDM system employed no interleaving, had an uncoded AOFDM target BER of $1 \%$, and used a WER threshold of $\alpha=10^{-2}$. It should be noted that the above BPS performance figures do not take into account the signaling overhead required for conveying the ARRNS/AOFDM modes, and hence constitute the upper-bound performance of the system. These performance figures suggest that the proposed ARRNS/AOFDM scheme outperforms all benchmarks in BPS terms for channel SNRs in excess of about $15 \mathrm{~dB}$ over the WATM channel of Fig. 1. Below $15 \mathrm{~dB}$, the turbo-convolutional code of [35] exhibits a higher BPS throughput. Our future work will be focused on incorporating turbo $\mathrm{BCH}$ codes in this system in an effort to further assess its performance potential in conjunction with AOFDM. Our further research will also invoke 


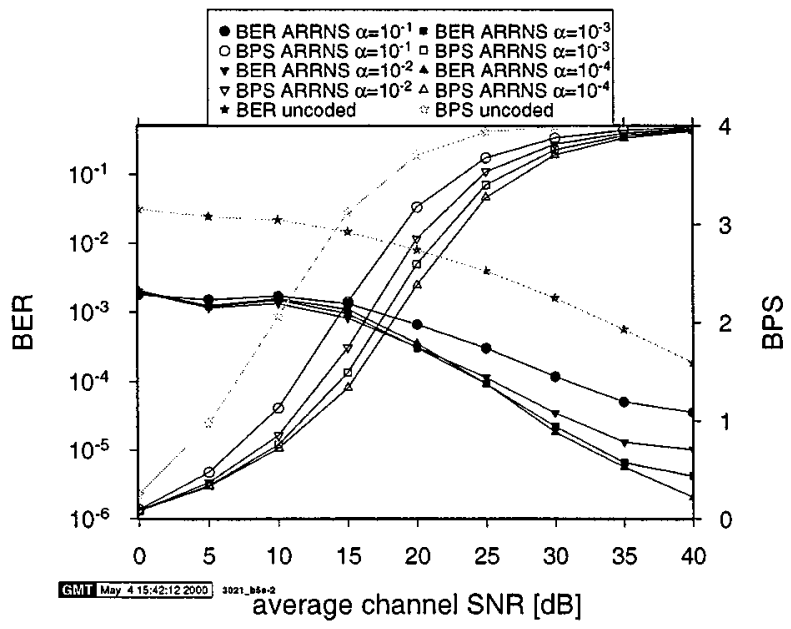

(a) target BER 5\% non-interleaved

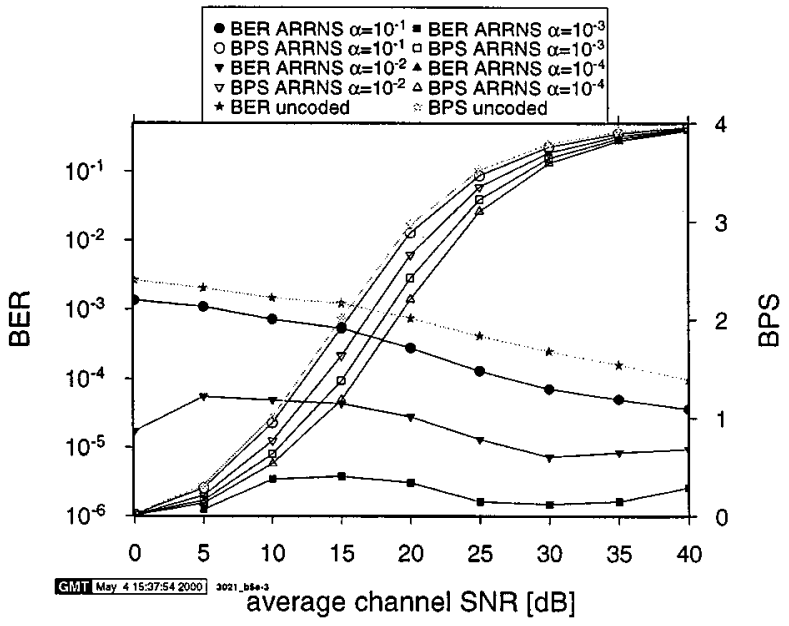

(b) target BER $0.5 \%$ non-interleaved

Fig. 8. BER and BPS throughput versus average channel SNR for soft-decision ARRNS-coded 512-subcarrier OFDM transmission employing adaptive modulation over the Rayleigh fading time-dispersive WATM channel of Fig. 1. (a) Uuncoded adaptive modulation target BER 5\%. (b) Uncoded target BER 0.5\% The stipulated WER values were $\alpha=10^{-1}, 10^{-2}, 10^{-3}$, and $10^{-4}$. The light grey curves show the uncoded BER and BPS throughput. Note that the data points of the BER $=0.5 \%$ curve associated with $\alpha=10^{-4}$ fell outside the plotted range and hence are invisible in the figure. The corresponding hard-decision based results were plotted in Fig. 7.

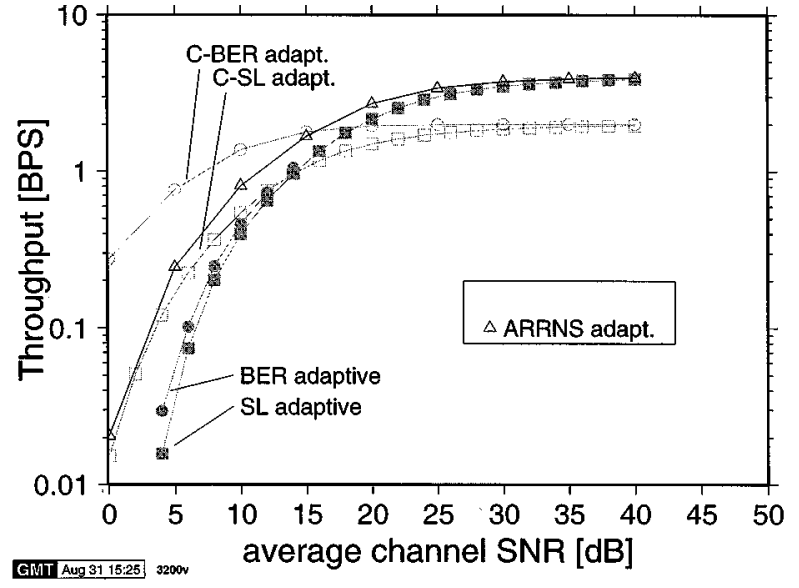

Fig. 9. BPS Throughput versus average channel SNR for AOFDM over the dispersive WATM channel of Fig. 1, for a maximum data bit error rate of $10^{-4}$. The lightly shaded curves present the performance of the variable throughput adaptive modulation schemes from [35] with and without convolutional turbo coding. The variable throughput systems include: convolutionally turbo coded (C-) and uncoded switching level adaptive (SL) and target-BER adaptive (BER) systems, as well as the joint adaptive RRNS/AOFDM system.

turbo trellis coding in conjunction with adaptive beam steering and interference cancellation.

\section{REFERENCES}

[1] R. Chang, "Synthesis of band-limited orthogonal signals for multichannel data transmission," Bell Syst. Tech. J., vol. 46, pp. 1775-1796, Dec. 1966.

[2] S. Weinstein and P. Ebert, "Data transmission by frequency division multiplexing using the discrete fourier transform," IEEE Trans. Commun. Technol., vol. COM-19, pp. 628-634, Oct. 1971.

[3] A. Peled and A. Ruiz, "Frequency domain data transmission using reduced computational complexity algorithms," in Proc. Int. Conf. Acoust., Speech, Signal Processing, ICASSP'80, vol. 3, Denver, CO, Apr. 9-11, 1980, pp. 964-967.
[4] B. Hirosaki, "An orthogonally multiplexed QAM system using the discrete fourier transform," IEEE Trans. Commun., vol. COM-29, pp. 983-989, July 1981.

[5] L. Cimini, "Analysis and simulation of a digital mobile channel using orthogonal frequency division multiplexing," IEEE Trans. Commun., vol. 33, pp. 665-675, July 1985.

[6] K. Kammeyer, U. Thisel, H. Schulze, and H. Bochmann, "Digital multicarrier transmission of audio signals over mobile radio channels," European Trans. Telecommun., vol. 3, pp. 243-253, May-June 1992.

[7] F. Mueller-Roemer, "Directions in audio broadcasting," J. Audio Eng. Soc., vol. 41, pp. 158-173, Mar. 1993.

[8] G. Plenge, "DAB - A new radio broadcasting system-State of development and ways for its introduction," Rundfunktech. Mitt., vol. 35, no. 2, 1991.

[9] M. Alard and R. Lassalle, "Principles of modulation and channel coding for digital broadcasting for mobile receivers," EBU Review, Tech. 224, pp. 47-69, Aug. 1987.

[10] Proc. 1st Int. Symp., DAB, Montreux, Switzerland, June 1992.

[11] I. Kalet, "The multitone channel," IEEE Trans. Commun., vol. 37, pp. 119-124, Feb. 1989.

[12] ETSI, , Digital Audio Broadcasting (DAB), 2nd ed: ETS 300 401, May 1997.

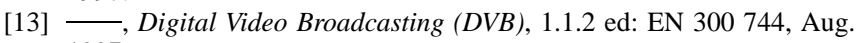
1997.

[14] _ CEPT Recommendation TIR 22-06, Harmonized Radio Frequency Bands for HIPERLAN Systems. Cedex, France: European Telecommunications Standards Institute, Sophia Antipolis, Jan. 1998.

[15] — ETSI TR 101 683, v0.1.2, Broadband Radio Access Networks (BRAN); High Performance Radio Local Area Networks (HIPERLAN) Type 2, System Overview. Cedex, France: European Telecommunications Standards Institute, Sophia Antipolis, 1999.

[16] K. Fazel and G. Fettweis, Eds., Multi-Carrier Spread-Spectrum. Norwell, MA: Kluwer, 1997, p. 260.

[17] L. Hanzo, W. Webb, and T. Keller, Single- and Multi-Carrier Quadrature Amplitude Modulation, New York: IEEE Press-Pentech Press, 2000.

[18] J. Davis and J. Jedwab, "Peak-to-mean power control in ofdm, golay complementary sequences, and Reed-Muller codes," IEEE Trans. Inform. Theory, vol. 45, pp. 2397-2417, Nov. 1999.

[19] H. Schmidt and K. Kammeyer, "Adaptive subträgerselektion zur Reduktion des Crest faktors bei OFDM," in 3. OFDM Fachgespräch in Braunschweig, 1998.

[20] R. Dinis and A. Gusmao, "Performance evaluation of OFDM transmission with conventional and 2-branch combining power amplification schemes," in Proc. IEEE Global Telecommun. Conf., Globecom 96, London, U.K., Nov. 18-22, pp. 734-739. 
[21] R. Dinis, P. Montezuma, and A. Gusmao, "Performance trade-offs with quasilinearly amplified OFDM through a 2-branch combining technique," in Proc. IEEE VTC'96, Atlanta, GA, pp. 899-903.

[22] R. Dinis, A. Gusmao, and J. Fernandes, "Adaptive transmission techniques for the mobile broadband system," in Proc. ACTS Mobile Commun. Summit '97, Aalborg, Denmark, Oct. 7-10, 1997, pp. $757-762$.

[23] B. Daneshrad, L. Cimini, Jr., and M. Carloni, "Clustered-OFDM transmitter implementation," in Proc. IEEE Int. Symp. Personal, Indoor, Mobile Radio Commun. (PIMRC'96), Taipei, Taiwan, Oct. 15-18, 1996, pp. 1064-1068.

[24] M. Okada, H. Nishijima, and S. Komaki, "A maximum likelihood decision based nonlinear distortion compensator for multicarrier modulated signals," IEICE Trans. Commun., vol. E81B, no. 4, pp. 737-744, 1998.

[25] R. Dinis and A. Gusmao, "Performance evaluation of a multicarrier modulation technique allowing strongly nonlinear amplification," in Proc. ICC 1998, pp. 791-796.

[26] P. Moose, "A technique for orthogonal frequency division multiplexing frequency offset correction," IEEE Trans. Commun., vol. 42, pp. 2908-2914, Oct. 1994.

[27] F. Daffara and O. Adami, "A new frequency detector for orthogonal multicarrier transmission techniques," in Proc. IEEE Veh. Technol. Conf. (VTC'95), Chicago, July 15-28, 1995, pp. 804-809.

[28] M. Sandell, J.-J. van de Beek, and P. Börjesson, "Timing and frequency synchronization in OFDM systems using the cyclic prefix," in Proc. Int Symp. Synchronization, Essen, Germany, Dec. 14-15, 1995, pp. 16-19.

[29] Y. Li and N. Sollenberger, "Interference suppression in OFDM systems using adaptive antenna arrays," in Proc. Globecom'98 Sydney, Australia, Nov. 8-12, 1998, pp. 213-218.

[30] - "Adaptive antenna arrays for OFDM systems with cochannel interference," IEEE Trans. Commun., vol. 47, pp. 217-229, Feb. 1999.

[31] Y. Li, L. Cimini, and N. Sollenberger, "Robust channel estimation for OFDM systems with rapid dispersive fading channels," IEEE Trans. Commun., vol. 46, pp. 902-915, Apr. 1998.

[32] C. Kim, S. Choi, and Y. Cho, "Adaptive beamforming for an OFDM sytem," in Proc. VTC'99 (Spring), Houston, TX, May 16-20, 1999.

[33] L. Lin, L. Cimini, Jr., and J.-I. Chuang, "Turbo codes for OFDM with antenna diversity," in Proc. VTC'99 (Spring), Houston, TX, May 16-20, 1999.

[34] M. Münster, T. Keller, and L. Hanzo, "Co-channel interference suppression assisted adaptive OFDM in interference limited environments," in Proc. VTC'99, pp. 284-288.

[35] T. Keller and L. Hanzo, "Blind-detection assisted sub-band adaptive turbo-coded OFDM schemes," in Proc. VTC'99 (Spring), Houston, TX, May 16-20, 1999, pp. 489-493.

[36] A. Czylwik, "Adaptive OFDM for wideband radio channels," in Proc. IEEE Global Telecommun. Conf., Globecom 96, pp. 713-718.

[37] P. Chow, J. Cioffi, and J. Bingham, "A practical discrete multitone transceiver loading algorithm for data transmission over spectrally shaped channels," IEEE Trans. Commun., vol. 48, pp. 772-775, 1995.

[38] T. Keller and L. Hanzo, "Adaptive orthogonal frequency division multiplexing schemes," in Proc. ACTS Mobile Commun. Summit'98, Rhodes, Greece, June 8-11, 1998, pp. 794-799.

[39] J. Torrance and L. Hanzo, "Latency and networking aspects of adaptive modems over slow indoors Rayleigh fading channels," IEEE Trans. Veh. Technol., vol. 48, pp. 1237-1251, 1998.

[40] W. Webb and L. Hanzo, Modern Quadrature Amplitude Modulation: Principles and Applications for Wireless Communications. New York: IEEE Press-Pentech Press, 1994.

[41] N. Szabo and R. Tanaka, Residue Arithmetic and Its Applications to Computer Technology. New York: McGraw-Hill, 1967.

[42] R. Watson and C. Hastings, "Self-checked computation using residue arithmetic," Proc. IEEE, vol. 54, pp. 1920-1931, Dec. 1966.

[43] W. Jenkins and B. Leon, "The use of residue number system in the design of finite impulse response filters," IEEE Trans. Circuits Syst., vol. CAS-24, pp. 191-201, Apr. 1977.

[44] M. Soderstrand, "A high-speed, low-cost, recursive digital filter using residue number arithmetic," Proc. IEEE, vol. 65, pp. 1065-1067, July 1977.

[45] M. Soderstrand and E. Fields, "Multipliers for residue number arithmetic digital filters," Electron. Lett., vol. 13, pp. 164-166, Mar. 1977.

[46] M. Soderstrand, W. Jenkins, and G. Jullien, Residue Number System Arithmetic: Modern Applications in Digital Signal Processing. New York: IEEE Press, 1986.

[47] E. Claudio, G. Orlandi, and F. Piazza, "A systolic redundant residue arithmetic error correction circuit," IEEE Trans. Computers, vol. 42, pp. 427-432, Apr. 1993.
[48] H. Krishna, K.-Y. Lin, and J.-D. Sun, "A coding theory approach to error control in redundant residue number systems-Part I: Theory and single error correction," IEEE Trans. Circuits Syst. II: Analog Digital Signal Processing, vol. 39, pp. 8-17, Jan. 1992.

[49] J.-D. Sun and H. Krishna, "A coding theory approach to error control in redundant residue number systems-Part II: Multiple error detection and correction," IEEE Trans. Circuits Syst. II: Analog Digital Signal Processing, vol. 39, pp. 18-34, Jan. 1992.

[50] T. Liew, L.-L. Yang, and L. Hanzo, "Soft-decision redundant residue number system based error correction coding," in Proc. VTC'99 (Fall), Amsterdam, Netherlands, Sept. 19-22, 1999, pp. 2974-2978

[51] L.-L. Yang and L. Hanzo, "Residue number system arithmetic assisted $m$-ary modulation," IEEE Commun. Lett., vol. 3, pp. 28-30, Feb. 1998

[52] - "Performance of residue number system based DS-CDMA over multipath fading channels using orthogonal sequences," ETT, vol. 9, pp. 525-536, Nov.-Dec. 1998.

[53] H. Krishna, K.-Y. Lin, and J.-D. Sun, "A coding theory approach to error control in redundant residue number systems-Part I: Theory and single error correction," IEEE Trans. Circuits Syst., vol. 39, pp. 8-17, Jan. 1992.

[54] H. Krishna and J.-D. Sun, "On theory and fast algorithms for error correction in residue number system product codes," IEEE Trans. Comput., vol. 42, pp. 840-852, July 1993.

[55] L.-L. Yang and L. Hanzo, "Coding theory and performance of redundant residue number system codes," IEEE Trans. Inform. Theory, submitted for publication.

[56] D. Chase, "A class of algorithms for decoding block codes with channel measurement information," IEEE Trans. Info. Theory, vol. IT-18, pp. 170-182, Jan. 1972.

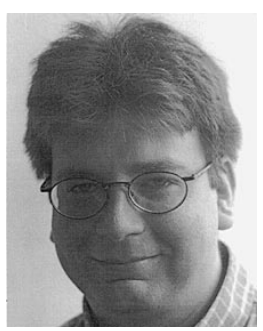

Thomas Keller studied electrical engineering at the University of Karlsruhe, Ecole Superieure d'Ingenieurs en Electronique et Electrotechnique, Paris, and the University of Southampton. He graduated with a Dipl.-Ing. degree in 1995. Between 1995 and 1999 he had been with the Wireless Multimedia Communications Group at the University of Southampton, where he completed the Ph.D. degree in mobile communications. His current areas of interest include adaptive OFDM transmission, wideband channel estimation, and error correction coding. He recently joined Ubinetics, Cambridge, U.K.

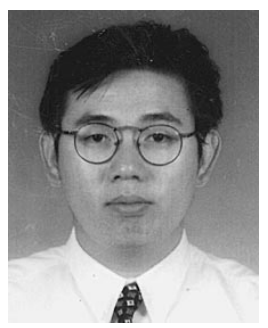

T. H. Liew received the B.Eng. degree in electronics engineering from the University of Southampton, U.K. Since 1997 he has been working toward the degree of Ph.D. in mobile communications at the University of Southampton, U.K. His current research interests are associated with coding and modulation for wireless channels. In this field he published a number of contributions.

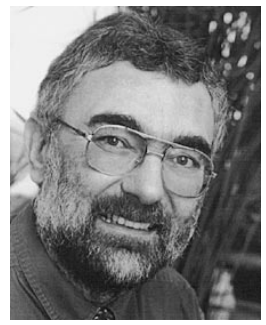

Lajos Hanzo (M'91-SM'92) graduated in electronics in 1976, and in 1983, he was conferred a Ph.D. During his 24-year career in telecommunications he has held various research and academic posts in Hungary, Germany and the U.K. Since 1986 he has been with the Department of Electronics and Computer Science, University of Southampton, U.K. and has been a Consultant to Multiple Access Communications Ltd., U.K. Currently he holds a Chair in telecommunications. He coauthored five books on mobile radio communications, coauthored over 300 research papers, organized and chaired conference sessions, presented overview lectures, and was awarded a number of distinctions. Currently he is managing an academic research team, working on a range of research projects in the field of wireless multimedia communications sponsored both by industry and under the auspices of the Engineering and Physical Sciences Research Council (EPSRC) U.K., the European IST Programme and the Mobile Virtual Centre of Excellence (VCE), U.K. For further information on research in progress and associated publications please refer to http://www-mobile.ecs.soton.ac.uk. 